Sensors 2005, 5, 604-612

sensors

ISSN 1424-8220

(C) 2005 by MDPI

http://www.mdpi.org/sensors

\title{
Determination of Atropine in Injection with $\beta$-Cyclodextrin Modified Ion Sensitive Field Effect Transistor Sensor
}

\author{
Xian-Wen $\mathrm{Li}^{1,2}$, Bo-Lun Yang ${ }^{1, *}$, Ying-Ying $\mathrm{Wu}^{1}$ and Hong-Ye Lin ${ }^{1}$ \\ ${ }^{1}$ Chemical Engineering Department, Xi'an Jiaotong University, Xi'an 710049, P.R. China \\ ${ }^{2}$ Science School, Guangdong Ocean University, Zhanjiang 524088, P.R. China \\ *Author to whom correspondence should be addressed. E-mail: blunyang@mail.xjtu.edu.cn
}

Received: 4 March 2005 / Accepted: 19 December 2005 / Published: 21 December 2005

\begin{abstract}
A new atropine drug-FET sensor, which was made of an ISFET (ion sensitive field effect transistor) and a drug sensitive membrane prepared by adding electric active matter to a $\beta$-cyclodextrin solution, was developed. The $\mathrm{pH}$ influence, selectivity, response curve, reproducibility, stability, response time and life of this sensor were investigated. A preliminary application of the sensor was discussed as well. The experimental results indicated that the sensor showed a Nernst response to atropine with a slope of 58.0 $\mathrm{mV} /$ decade over the concentration range of $5.0 \times 10^{-3}-1.0 \times 10^{-6} \mathrm{~mol} / \mathrm{L}$ and the $\mathrm{pH}$ range of $5.0-8.5$. The sensor detection limit was $8.0 \times 10^{-7} \mathrm{~mol} / \mathrm{L}$. The performance of this sensor was very stable when it was used to determine atropine concentration in a medicinal injection. The obtained results agreed well with the pharmacopoeia method.
\end{abstract}

Keywords: atropine, $\beta$-cyclodextrin, ion sensitive field effect transistor, drug analysis.

\section{Introduction}

Since the ion sensitive field effect transistor (ISFET) response to $\mathrm{H}^{+}$was firstly reported by $\mathrm{P}$. Bergved [1], it has drawn the attention of many scientists because of the excellent characteristics of its solid state, miniature size and highly potential value. Furthermore, the ISFET can be microminiaturized, integrated and multi-functional. Owing to the peculiarities of the ISFET, such as self-impedance alteration, lower output impedance and compatibility with MOS processing technology, scientists can utilize it to prepare new kinds of intelligent analysis instruments by assembling the sensor and the determining circuit. In recent years, there have been great advances in the ISFET researches. By substituting different sensitive membranes for the MOSFET metal grid, many kinds of 
ISFET sensors have been developed. These sensors of new types can be used in many fields such as chemistry, biology, medicine, environment protection, and industrial control [2-5].

As a drug resistant to choline, atropine has been widely used to cure some diseases. On one hand, it can treat muscle spasm, inhibit gland secretion, dilate eye pupils, and excite the respiratory central nerve. On the other hand, it can assist serious patients in shock caused by infection or poisoning by organic phosphorus farm chemicals. It can also relieve the pains caused by gastroenteric spasm, kidney angina and gallbladder angina. Since atropine is a poisonous drug in itself, its dosage must be strictly controlled, and its clinical determination is significant. The research of an ISFET chemical sensor, therefore, has fine prospects.

The measurements of atropine, including UV spectroscopy and HPLC methods, however, have some disadvantages [6], such as their complex equipment, troublesome operations, etc. Therefore, two methods for measuring atropine with ISFET have been reported. One was based on tri-o-e- $\beta$-CD, which is complicated in structure and difficult to be synthesized [7], another was based on sodium tetraphenylboron, as the electric active material. Both of them allowed only narrow ranges of $\mathrm{pH}$, high lower-limit of the measurements [8].

In this research, a new detection method for atropine based on ISFET was studied and a new-type atropine ISFET sensor was developed. Comparing with what has been done during the past, $\beta$-CD was used as its electric active material herein, which is much easier to be obtained. It allowed wider $\mathrm{pH}$ ranges and possessed higher sensitivity.

To confirm the validity of this method, several operating factors such as the $\mathrm{pH}$, selectivity, response curve, reproducibility, stability, response time and the life of this sensor was investigated. The preliminary application of the sensor is reported in this paper.

\section{Experiments}

\subsection{Instruments and reagents}

The instruments used in the experiments include ISFET determination meter (XG-ISFET-02, made in China), pHS-3C precision acidometer (produced by Shanghai Analytical Instrument Factory, China), and Type-217 mercurous chloride electrode (produced by Shanghai Light and Electrical Instrument Factory, China).

The reagents used in the experiments include $\beta$-CD (A.R), poly vinyl chloride (PVC) powder (purchased from Shanghai Chemical Factory), dibuty (o-) phthalate (A.R), and Atropine (A.R). Other reagents are of analytical grade and double distilled water used in all the experiments.

\subsection{The preparation of atropine sensitive field effect transistor}

Based on the plane technology, the n-channel depletion mode field effect transistor (FET) was prepared by depositing a layer of insulating material of $\mathrm{Si}_{3} \mathrm{~N}_{4} / \mathrm{SiO}_{2}$ on the crystal face (100) of p-type silicon with the conductivity of $6 \sim 8 \mathrm{~S} / \mathrm{cm}$ [9].

The FET was isolated in a plastic or glass pipe with a sealing material (silicon rubber or epoxy resin), two metal fibres were drawn from the source (S) and drain (D), and the $\mathrm{Si}_{3} \mathrm{~N}_{4} / \mathrm{SiO}_{2}$ layer of the 
insulated-gate was exposed. A layer of active membrane was painted on the $\mathrm{Si}_{3} \mathrm{~N}_{4}$ surface. The preparation process of atropine sensitive Drug-FET was as follows: a suspension was firstly prepared by adding $500 \mathrm{mg}$ of PVC as base polymer, $200 \mathrm{mg}$ dibuty(o-)phthalate as plasticizer, $8 \mathrm{mg} \beta-\mathrm{CD}$ as receptor, and $5 \mathrm{ml}$ of tetrahydrofuran as solvent respectively. The suspension was then smeared on the surface of the $\mathrm{Si}_{3} \mathrm{~N}_{4} / \mathrm{SiO}_{2}$ gate of the FET and dried at room temperature for 24 hours. Finally, the atropine sensitive drug-FET was activated by immerging it in the $1.0 \times 10^{-3} \mathrm{~mol} / \mathrm{L}$ atropine solution for ten hours, so that the FET could respond to Atropine. The structure of ISFET is shown in Figure 1.

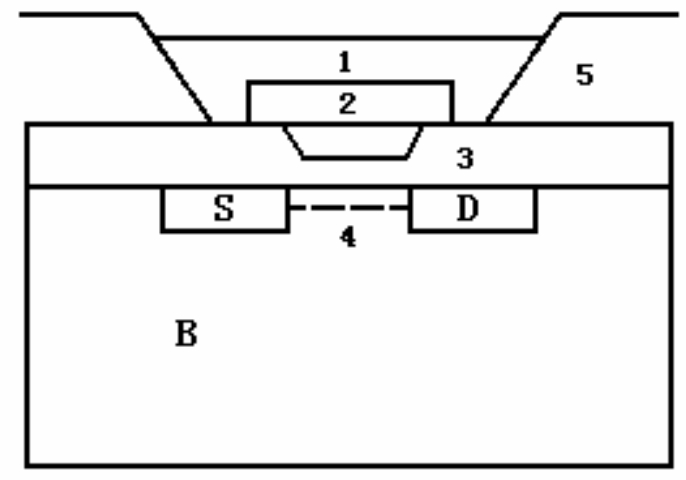

Figure 1. Structure of ISFET. S: source; D: drain; B: p-silicon; 1- drug sensitive membrane; 2 - $\mathrm{Si}_{3} \mathrm{~N}_{4} ; 3$ - $\mathrm{SiO}_{2} ; 4$ - n-channel; 5 - silicone rubber.

\subsection{Measurement of the sensor's performances}

The atropine ion sensitive FET chemical sensor was prepared by connecting the atropine sensitive drug-FET and a mercurous chloride electrode with a double salt-bridge (an outer salt bridge in a solution of $1.0 \mathrm{~mol} / \mathrm{LKNO}_{3}$ ) to become a loop. When this sensor was used, the voltage $\mathrm{V}_{\mathrm{DS}}$ and the current $\mathrm{I}_{\mathrm{DS}}$ between the source (S) and the drain (D) should be kept constant. The relationship between the output voltage $\left(\mathrm{V}_{\mathrm{Gout}}\right)$ of the gate $(\mathrm{G})$ and the concentration of ions obeyed the Nernst equation. The working curve could be obtained by drawing the $\mathrm{V}_{\mathrm{Gout}}$ and $\mathrm{pC}$ (minus logarithm of the concentration of Atropine) [10-11].

If the current $I_{D S}$ and voltage $V_{D S}$ between drain (D) and source (S) are kept constant, the voltage of gate (G) $V_{\text {Gout }}$ varies with the change of the component concentrations to be determined. The relationship between them is in accordance with the Nernst formula as shown below:

$$
\mathrm{E}_{\mathrm{M}}=(\mathrm{RT} / \mathrm{ZiF}) \ln \mathrm{a}_{\mathrm{i}}
$$

Where, $a_{i}$ is the activity of atropine.

In this case, the sensor's gate voltage $\mathrm{V}_{\text {Gout }}$ was the algebra sum of the reference electrode potential

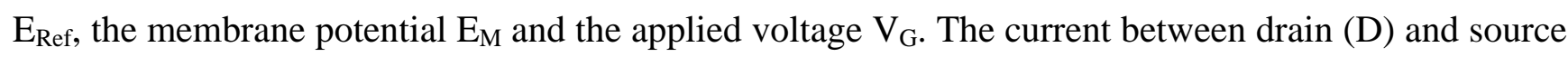
(S) is shown as follows:

$$
\mathrm{I}_{\mathrm{DS}}=\mathrm{K}\left[\left(\mathrm{V}_{\mathrm{G}}+\mathrm{E}_{\mathrm{M}}-\mathrm{V}_{\mathrm{T}}\right)-\mathrm{V}_{\mathrm{DS}} / 2\right]
$$

Where, $\mathrm{K}$ is a parameter related to the sensor, and $\mathrm{V}_{\mathrm{T}}$ is the grid voltage related to the performance of sensor and the reference electrode potential $E_{\text {Ref. }} I n$ this case:

$$
\mathrm{I}_{\mathrm{DS}}=0, \mathrm{~V}_{\mathrm{DS}}=0 \text {, }
$$


Introducing Eq. (3) into Eq. (2) and arranging, we obtain

$$
\triangle \mathrm{V}_{\mathrm{Gout}}=\mathrm{E}_{\mathrm{M}}=(\mathrm{RT} / \mathrm{ZiF}) \ln \mathrm{a}_{\mathrm{i}}
$$

During the determination, the ISFET and reference electrode (Type-217 mercurous chloride electrode) formed a measuring current loop. An Operational amplifier was used to bring a stable criterion voltage. The operating state $\left(\mathrm{V}_{\mathrm{DS}}, \mathrm{I}_{\mathrm{DS}}\right)$ of ISFET was calibrated by adjusting $\mathrm{W}_{1}$ and $\mathrm{W}_{2}$. When the concentration of solution varied, the $\mathrm{V}_{\text {Gout }}$ also altered. The relative curve of $\mathrm{V}_{\text {Gout }}$ versus-log $\mathrm{C}$ (C was the concentration of solution to be determined) could be obtained according to the experimental results. The determination principle of atropine ISFET sensor is shown in Figure 2.

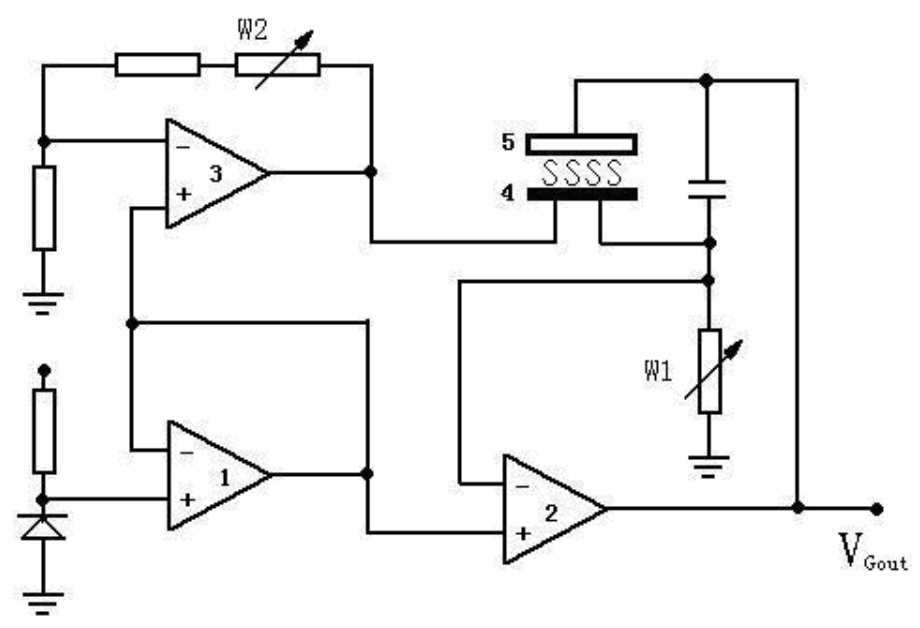

Figure 2. Determination principle of ISFET.

\section{Results and Discussion}

\subsection{The influence of the $\beta$-CD content}

The sensitivity of measurement is greatly influenced by the content of $\beta$-CD, which can be used as the electro-active substance responding to atropine, the response between the $\mathrm{V}_{\text {Gout }}$ with content of $\beta$-CD thus was investigated and the results are shown in Table 1. It can be known from the results that the maximum response appears when the $\beta$-CD content was $8 \mathrm{mg}$ in the membrane.

Table 1. The influence of the $\beta$-CD content.

\begin{tabular}{cccc}
\hline Membrane constituents & PVC $(\mathrm{mg})$ & $\beta$-CD $(\mathrm{mg})$ & $\mathrm{V}_{\text {Gout }}(\mathrm{mV})$ \\
\hline 1 & 500 & 4 & 40.5 \\
2 & 500 & 6 & 51.7 \\
3 & 500 & 8 & 58.1 \\
4 & 500 & 10 & 55.2 \\
\hline
\end{tabular}




\subsection{The effects of $p H$}

Sets of solutions were prepared by mixing $1.0 \times 10^{-3} \mathrm{~mol} / \mathrm{L}$ atropine solutions, $1.0 \mathrm{~mol} / \mathrm{L} \mathrm{HCl}$ and 1.0 $\mathrm{mol} / \mathrm{L} \mathrm{NaOH}$. The $\mathrm{pHs}$ of the mixture solutions were in a range from 3.0 to 9.0. The effect of $\mathrm{pH}$ on $\mathrm{V}_{\text {Gout }}$ was investigated and the results are shown in Figure 3 . The figure showed that the $\mathrm{V}_{\text {Gout }}$ did not change significantly in the $\mathrm{pH}$ range from 5.0 to 8.5, however, as a weak base at the low side of $\mathrm{pH}$, atropine could be combined with acid into salt when the acidity was increased, the effective concentration of atropine was decreased, the response $\mathrm{V}_{\text {Gout }}$ thus was decreased. When the basicity in the solution became stronger, however, $\beta$-CD could change in structure as electric active matter, thus leading to augmentation of the response $\mathrm{V}_{\mathrm{Gout}}$.

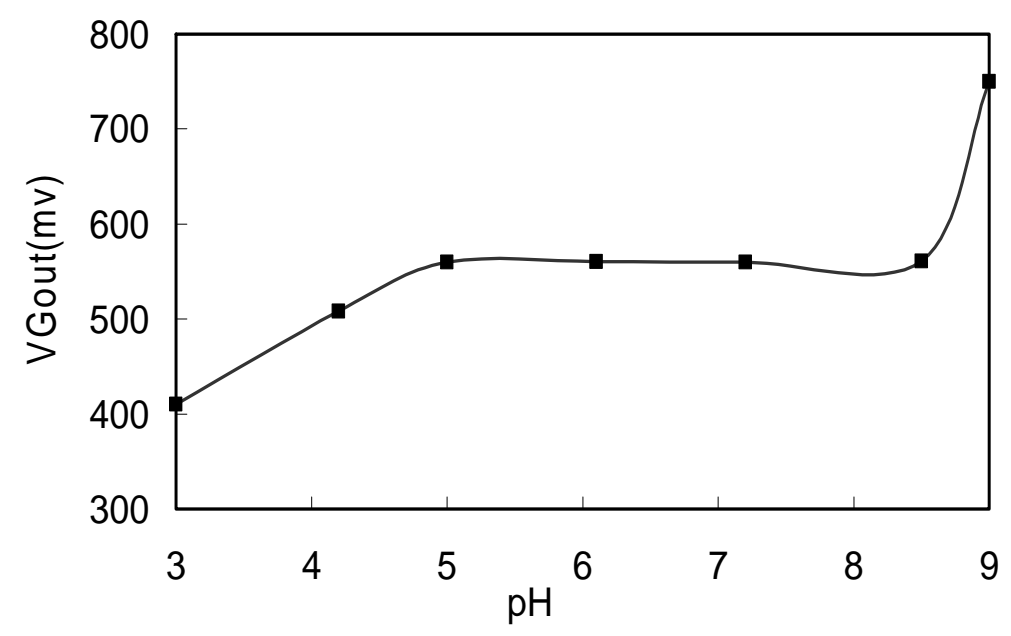

Figure 3. The effects of $\mathrm{pH}$.

\subsection{The selectivity of the sensor}

Some chemicals were chosen as the research objects. All of them are the substances that would be met during the application of the sensor such as producing and clinic using processes of atropine, or substances that would affect the determination process of atropine.

Based on the method of fixation disturbing for mixing solution, the selectivity coefficient of the sensor was determined. The results are shown in Table 2. It showed that the sensor had high selectivity since most of the substances did not disturb the determination of atropine except for procaine.

\subsection{The response curve of the sensors}

Since the response of the sensor used in this work is a kind of host-guest response between the $\beta$-CD and atropine, $\mathrm{V}_{\mathrm{Gout}}$ of the sensor and the concentration of the atropine showed a linear relationship as given in Figure 4. 
Table 2. The selective coefficients of the sensor (solution-mixing method).

\begin{tabular}{cc}
\hline Disturbing substance & $K$ \\
\hline $\mathrm{KCl}$ & $1.2 \times 10^{-3}$ \\
$\mathrm{NaCl}$ & $5.0 \times 10^{-4}$ \\
$\mathrm{NH}_{4} \mathrm{Cl}$ & $1.5 \times 10^{-3}$ \\
$\mathrm{Ca}\left(\mathrm{NO}_{3}\right)_{2}$ & $3.0 \times 10^{-3}$ \\
Glucose & $1.5 \times 10^{-3}$ \\
Procaine & Interfered \\
Caffeine & $1.1 \times 10^{-2}$ \\
$\mathrm{VB}_{1}$ & $1.5 \times 10^{-2}$ \\
Erythromycin & $3.5 \times 10^{-2}$ \\
Adrenaline & $5.0 \times 10^{-2}$ \\
\hline
\end{tabular}

In the concentration range of $5.0 \times 10^{-3}-1.0 \times 10^{-6} \mathrm{~mol} / \mathrm{L}$, the relationship between $\mathrm{V}_{\mathrm{Gout}}$ and $\mathrm{pC}$ obeyed the linear correlation equation: $\mathrm{V}_{\mathrm{Gout}}(\mathrm{mV})=518.5+58.0 \mathrm{pC}(\mathrm{mol} / \mathrm{L})$. The linear correlation coefficient of the equation $\gamma=0.999$. The sensors detection limit was $8.0 \times 10^{-7} \mathrm{~mol} / \mathrm{L}$, while the RSD of atropine solution determination for five times was $2.1 \%$.

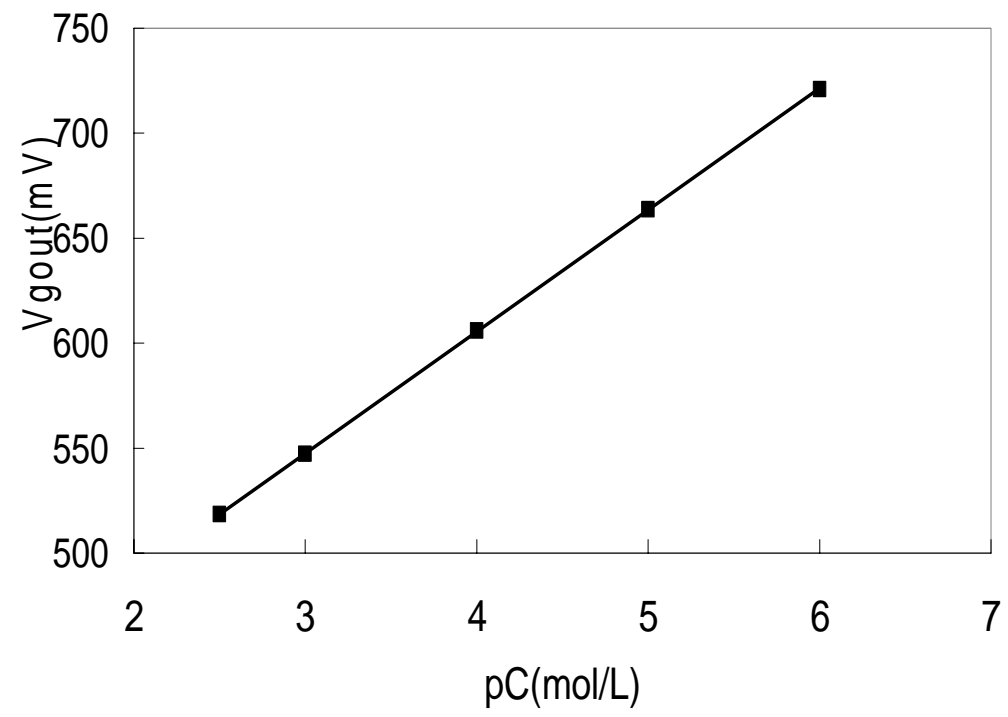

Figure 4. Calibration of curve.

\subsection{The reproducibility}

In the concentration range of $1.0 \times 10^{-3}-1.0 \times 10^{-6} \mathrm{~mol} / \mathrm{L}$ atropine solutions, each sample was repeatedly determined for five times, the variation of $\mathrm{V}_{\text {Gout }}$ is shown in Table 3. For each sample, the change of the $\mathrm{V}_{\text {Gout }}$ was less than $1 \mathrm{mV}$. When the sensor was continuously used for four hours, the $\mathrm{V}_{\text {Gout }}$ was still less than $1 \mathrm{mV}$. Therefore, it can be considered that the reproducibility of the sensors is good and the drift is small. 
Table 3. Reproducibility of the sensor.

\begin{tabular}{cccccccc}
\hline $\begin{array}{c}\text { Atropine } \\
(\mathrm{mol} / \mathrm{L})\end{array}$ & \multicolumn{5}{c}{$\mathrm{V}_{\text {Gout }}(\mathrm{mV})$} & Average & Reproducibility \\
\hline $1.0 \times 10^{-6}$ & 847 & 848 & 846 & 847 & 848 & 847.2 & $\leqslant \pm 1.0$ \\
$1.0 \times 10^{-5}$ & 787 & 787 & 786 & 787 & 788 & 787.0 & $\leqslant \pm 1.0$ \\
$1.0 \times 10^{-4}$ & 729 & 728 & 727 & 728 & 728 & 728.0 & $\leqslant \pm 1.0$ \\
$1.0 \times 10^{-3}$ & 670 & 671 & 670 & 671 & 671 & 670.6 & $\leqslant \pm 1.0$ \\
\hline
\end{tabular}

3.6. The response time and the life of the sensors

The response time and the life of the sensors are also important factor. Generally, the response time is defined as the time interval from the beginning of determining until the change of the $\mathrm{V}_{\text {Gout }}$ is less than $1 \mathrm{mV}$. The life of the sensor is the time interval from the start using until it cannot response normally.

In this work, the response time was less than 30 seconds for $5.0 \times 10^{-3}-1.0 \times 10^{-6} \mathrm{~mol} / \mathrm{L}$ atropine solutions, and the test on the life of the sensor showed that the sensor still remained good response characteristics after three months storage.

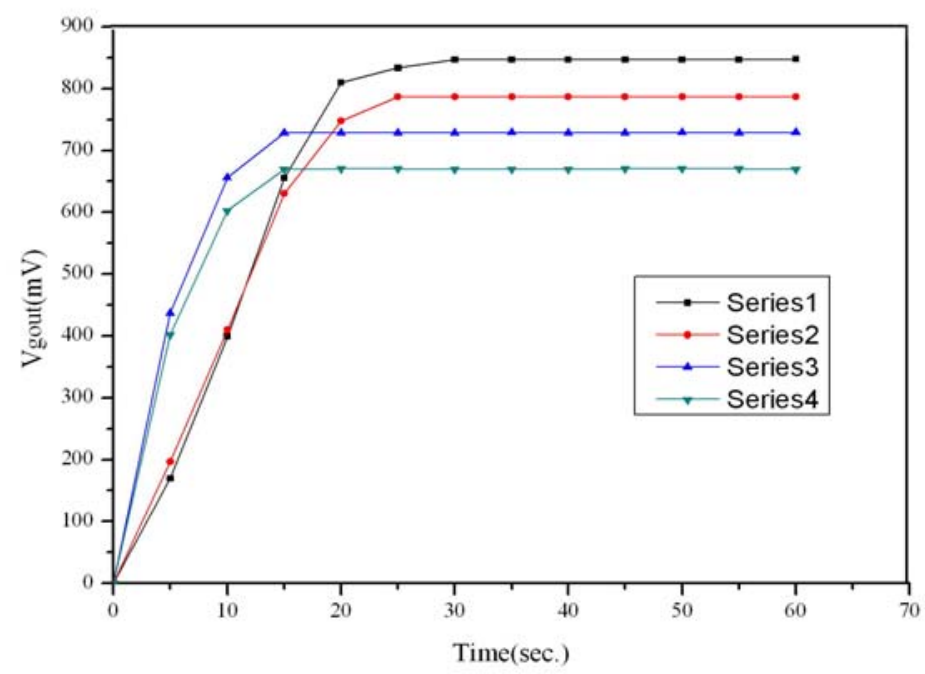

Figure 5. Response time of sensor. Series1, $1.0 \times 10^{-6} \mathrm{~mol} / \mathrm{L}$ atropine, Series2, $1.0 \times 10^{-5} \mathrm{~mol} / \mathrm{L}$ atropine, Series, $31.0 \times 10^{-4} \mathrm{~mol} / \mathrm{L}$ atropine, Series $4,1.0 \times 10^{-3} \mathrm{~mol} / \mathrm{L}$ atropine.

\subsection{The preliminary application of the sensors}

This sensor was applied to quantitatively analyze injection atropine. The standard curve was firstly obtained: the atropine sample was weighed to prepare some reserve solution in the concentration of $1.0 \times 10^{-3} \mathrm{~mol} / \mathrm{L}$, this solution was diluted step by step and a series of standard solutions were obtained. The $\mathrm{V}_{\text {Gout }}$ of these solutions were measured respectively, and a plot of $\mathrm{V}_{\text {Gout }}$ with minus logarithm of 
the concentration for standard sample thus was obtained. When the $\mathrm{V}_{\mathrm{Gout}}$ of the sensor was measured in the same condition, the amount of injection atropine thus was known from the standard curve.

This method was also compared with the spectroscopy method in Pharmacopoeia of P. R. China [12]. The results are shown in Table 4. It showed that the results obtained by this work agreed well with the data reported by the traditional method. However, since the proposed method allows wider $\mathrm{pH}$ ranges, possesses higher sensitivity, accuracy, rapidness, easy operation and the characteristic of the effective determination without using any expensive equipment, it is more perspective.

Table 4. The determination of atropine concentration for an injection.

\begin{tabular}{ccccccc}
\hline Method/No & 1 & 2 & 3 & 4 & 5 & $\mathrm{X} \pm \mathrm{SD}$ \\
\hline Sensor & 1.02 & 1.00 & 1.02 & 1.06 & 1.08 & $1.04 \pm 0.03$ \\
Pharmacopoeia & 1.05 & 1.03 & 1.06 & 1.04 & 1.06 & $1.05 \pm 0.01$ \\
\hline
\end{tabular}

\section{Conclusion}

A new ISFET sensor modified with $\beta$-CD for determining atropine concentrations in solutions was developed._The determination results obtained by using the new ISFET sensor agreed well with the data obtained by pharmacopoeia method.

Since the sensor is a kind of host-guest response between $\beta-C D$ and atropine, it shows a high sensitivity. Meanwhile, because of the sensor's peculiarities of self-impedance alteration, lower output impedance and compatibility with MOS processing technology, it can be beneficially used to prepare a new kind of intelligent analysis instrument. The analytical method proposed in this research provides many advantages such as full-solid, miniature volume, the potential of being microminiaturized, integrated and multi-functional capability.

\section{References}

1. Bergveld, P. Development of an ion-sensitive solid-state device for neurophysiological measurements. IEEE Trans. Biomed. Eng. 1970, BM17, 70.

2. Bratov, A.; Abramova, N.; Dominguez, C. Investigation of chloride sensitive ISFETs with different membrane compositions suitable for medical applications. Anal. Chim. Acta. 2004, 514, 99-106.

3. Luo, X.L.; Xu, J.J.; Zhao, W.; Chen, H.Y. Ascorbic acid sensor based on ion-sensitive field-effect transistor modified with $\mathrm{MnO}_{2}$ nanoparticles. Anal. Chim. Acta. 2004, 512, 57-61.

4. Pogorelova, S.P.; Kharitonov, A.B.; Willner, I.; Sukenik, C.N.; Pizem, H.; Bayer, T. Development of ion-sensitive field-effect transistor-based sensors for benzylphosphonic acids and thiophenols using molecularly imprinted $\mathrm{TiO}_{2}$ films. Anal. Chim. Acta. 2004, 504, 113-122.

5. Bergveld, P. Thirty years of ISFET - what happened in the past 30 years and what may happen in the next 30 years. Sensor. Actuat. B-Chem. 2003, 88, 1-20.

6. Lin, H.Q.; Li, J. Determination of atropine in tablet by HPLC. Guangdong Pharm. J. 2004, 14, 18-19. 
7. Li, X.W.; Yang, B.L. Study on tri-o-e- $\beta-C D$ modified ISFET and its application. Chem. Sens. 2001, 17(Suppl. B), 402-404.

8. Wang, D.W.; Li, X.W.; Liu, Y.Q. Study on the determination of atropine by ISFET chemical sensor. J. Anal. Sci. (Wuhan). 2002, 18, 237-239.

9. Li, X.W.; Huang, Q. Development and application of silicotungstic acid-berberine field effcet transistor. Chin. J. Anal. Chem. 1997, 25, 1297-1299.

10. Li, X.W.; Yang, B.L. An ion field effect transistor sensor for the determinnation of dioxopromethazine. Chin. J. Anal. Chem. 2003, 31, 896.

11. Liu, Y.Q.; Li, X.W. Study on ephedrine sensor by ion sensitive field effect transistor. Proceedings of the 8th International Meeting on Chemical Sensor. Basel, Switzerland. 2000, 419-421.

12. State pharmacopoeia commission. Chinese Pharmacopoeia (II). Beijing: Chem. Industry Press. 2005, 911.

(C) 2005 by MDPI (http://www.mdpi.org). Reproduction is permitted for noncommercial purposes. 